\title{
Secondary frequency regulation in power networks with on-off load side participation
}

\author{
Andreas Kasis, Nima Monshizadeh and Ioannis Lestas
}

\begin{abstract}
We study the problem of secondary frequency regulation where ancillary services are provided via load-side participation. In particular, we consider on-off loads that switch when prescribed frequency thresholds are exceeded in order to assist existing secondary frequency control mechanisms. We show that system stability is not compromised despite the switching nature of the loads. However, such control policies are prone to Zeno-like behavior, which limits the practicality of these schemes. As a remedy to this problem, we propose a hysteresis on-off policy and provide stability guarantees in this setting. We provide numerical investigations of the results on a realistic power network.
\end{abstract}

\section{INTRODUCTION}

It is anticipated that renewable sources of generation will increase their penetration in power networks in the near future [1], [2]. This is expected to introduce intermittency in the power generated resulting in additional challenges in the real time operation of power networks that need to be addressed.

A main objective in the operation of a power system is to ensure that generation matches demand in real time. This is achieved by means of primary and secondary frequency control schemes with the latter also ensuring that the frequency returns to its nominal value $(50 \mathrm{~Hz}$ or $60 \mathrm{~Hz})$. Secondary frequency control is traditionally performed by having the generation side following demand [3]. However, a large penetration of renewable sources of generation limits the controllability of generation and at the same time makes the system more sensitive to disturbances due to the reduced system inertia [4]. Controllable loads are considered by many a promising solution to counterbalance intermittent generation, being able to adapt their demand based on frequency deviations, providing fast response at urgencies. Recently, various research studies focused on the inclusion of controllable demand to aid both primary control as in [5]-[9] and secondary control as in [10], [11], [12].

Further from providing ancillary services at urgencies, it is also desired that controllable loads are non-disruptive, i.e. their assistance should have a negligible effect on users comfort, see e.g. [13]. Non-disruptive load-side control schemes ensure that loads alter their demand at urgencies but return to their normal operation when the danger for the network has been surpassed. Moreover, in many occasions, a realistic representation of loads involves only a discrete set of possible demand values, e.g. on and off states. Hence, incorporating on-off controllable loads that appropriately react to frequency

This work was supported by ERC starting grant 679774. Andreas Kasis, Nima Monshizadeh and Ioannis Lestas are with the Department of Engineering, University of Cambridge, Trumpington Street, Cambridge, CB2 1PZ, UK; e-mails: ak647@cam.ac.uk, n.monshizadeh@eng.cam.ac.uk icl20@cam.ac.uk. deviations in power networks is of particular interest in loadside participation schemes.

In this paper, we consider controllable on-off loads that switch when some frequency deviation is reached so that they assist the network at urgencies (i.e. when large frequency deviations are experienced) and otherwise return to their original operation. It will be shown that the inclusion of such loads does not compromise the stability of the power network, and result in enhanced frequency performance. However, it will be observed that such controllable loads may switch arbitrarily fast within a finite interval of time, or in other words, exhibit Zeno behavior. To avoid this, we propose on-off loads with hysteretic dynamics. Stability guarantees are again provided for this class of loads, and the absence of the Zeno phenomenon is analytically proven. We provide a numerical validation of our results through a simulation on the IEEE New York/ New England 68-bus system.

The structure of the paper is as follows: Section II includes some basic notation and preliminaries and in section III we present the power network model. In section IV we consider controllable demand that switches on/off whenever certain frequency thresholds are met and present our results concerning network stability. In section $\mathrm{V}$, we consider controllable loads with hysteretic patterns and show that stability results extend to this case. We demonstrate our results through a simulation on the IEEE New York/ New England 68-bus system in section VI. Finally, conclusions are drawn in section VII. The proofs of the results can be found in [14], which is an extended version of this paper.

\section{NOTATION}

Real numbers are denoted by $\mathbb{R}$, and the set of $n$ dimensional vectors with real entries is denoted by $\mathbb{R}^{n}$. The set of natural numbers, including zero, is denoted by $\mathbb{N}_{0}$. We use $\mathbf{0}_{n}$ to denote $n \times 1$ vector with all elements equal to 0 . For a discrete set $\Sigma$, let $|\Sigma|$ denote its cardinality. For a set $A$, let $\overline{c o}(A)$ denote its convex closure. For a point $x \in R^{n}$ and positive constant $\delta$ let $B(x, \delta)$ denote the ball of radius $\delta$ around $x$. Moreover, let $\mathcal{B}\left(\mathbb{R}^{n}\right)$ denote the collection of subsets of $\mathbb{R}^{n}$.

We use the notions of Lebesgue measurable set, zero measure set and Lebesgue measurable function from [15]. Furthermore, for a Lebesgue measurable set $A$, let $\mu(A)$ denote its Lebesgue measure. For notation convenience, Lebesgue measures will be referred as just measures. When something holds almost everywhere within a measurable set $A$, it means that it holds everywhere in $A$ except on sets $N$ satisfying $N \subset A: \mu(N)=0$. For a set $A$ and scalar $b$, $A \leq b$ denotes that all elements in $A$ are less than or equal to $b$. Moreover, the function $\operatorname{sgn}(x)$ takes a value of 1 when $x$ is non-negative and -1 otherwise. 
Within the paper a class of switching systems will be considered and the notion of Filippov solutions will be used for their analysis (see e.g. [16]). In particular, for any, potentially discontinuous, function $X: \mathbb{R}^{n} \rightarrow \mathbb{R}^{n}$, the Filippov set valued map $F[X]: \mathbb{R}^{n} \rightarrow \mathcal{B}\left(\mathbb{R}^{n}\right)$ is defined as,

$$
F[X](x) \equiv \bigcap_{\delta>0} \bigcap_{\mu(S)=0} \overline{c o}(X(B(x, \delta) \backslash S)), x \in \mathbb{R}^{n}
$$

where $\bigcap_{\mu(S)=0}$ denotes the intersection over all sets $S$ of Lebesgue measure zero.

In order to facilitate the analysis of differential equations with discontinuous vector fields $X: \mathbb{R}^{n} \rightarrow \mathbb{R}^{n}$, the dynamical system below (a differential inclusion) is often considered

$$
\dot{x}(t) \in F[X](x(t)) .
$$

For systems described by (2), a Filippov solution is defined as an absolutely continuous map $x:\left[0, t_{1}\right] \rightarrow \mathbb{R}^{n}$ that satisfies (2) for almost all $t \in\left[0, t_{1}\right]$. For the system that will be studied in the paper we will show that Filippov solutions exist and are unique.

Remark 1: Note that the use of Filippov solutions allows the study of systems with discontinuous dynamics when there are infinitely many switches at finite time, a phenomenon known as Zeno behavior.

\section{NETWORK MODEL}

We describe the power network model by a connected graph $(N, E)$ where $N=\{1,2, \ldots,|N|\}$ is the set of buses and $E \subseteq N \times N$ the set of transmission lines connecting the buses. Furthermore, we use $(i, j)$ to denote the link connecting buses $i$ and $j$ and assume that the graph $(N, E)$ is directed with arbitrary orientation, so that if $(i, j) \in E$ then $(j, i) \notin E$. For each $j \in N$, we use $i: i \rightarrow j$ and $k: j \rightarrow k$ to denote the sets of buses that are predecessors and successors of bus $j$ respectively. It is important to note that the form of the dynamics in (3)-(4) below is unaltered by any change in the graph ordering, and all of our results are independent of the choice of direction. The following assumptions are made for the network:

1) Bus voltage magnitudes are $\left|V_{j}\right|=1$ p.u. for all $j \in N$.

2) Lines $(i, j) \in E$ are lossless and characterized by their susceptances $B_{i j}=B_{j i}>0$.

3) Reactive power flows do not affect bus voltage phase angles and frequencies.

We use swing equations to describe the rate of change of frequency at generation buses, while power must be conserved at each of the load buses. This motivates the following system dynamics (e.g. [17]),

$$
\begin{gathered}
\dot{\eta}_{i j}=\omega_{i}-\omega_{j},(i, j) \in E, \\
M_{j} \dot{\omega}_{j}=-p_{j}^{L}+p_{j}^{M}-\left(d_{j}^{c}+d_{j}^{u}\right)-\sum_{k: j \rightarrow k} p_{j k}+\sum_{i: i \rightarrow j} p_{i j}, j \in N, \\
p_{i j}=B_{i j} \sin \eta_{i j}-p_{i j}^{n o m},(i, j) \in E .
\end{gathered}
$$

In system (3) the time-dependent variables $p_{j}^{M}, \omega_{j}$ and $d_{j}^{c}$ represent, respectively, deviations from a nominal value ${ }^{1}$

\footnotetext{
${ }^{1} \mathrm{~A}$ nominal value is defined as an equilibrium of (3) with frequency equal to $50 \mathrm{~Hz}$ (or $60 \mathrm{~Hz}$ ).
}

of the mechanical power injection, and the frequency and controllable load present at bus $j$. The quantity $d_{j}^{u}$ is also a time-dependent variable that represents the uncontrollable frequency-dependent load and generation damping present at bus $j$. Furthermore, the quantities $\eta_{i j}$ and $p_{i j}$ are timedependent variables that represent, respectively, the power angle difference, and the deviation from the nominal value, $p_{i j}^{n o m}$, of the power transmitted from bus $i$ to bus $j$. The constant $M_{j}>0$ denotes the generator inertia. We study the response of system (3) at a step change in the uncontrollable demand $p_{j}^{L}$ at each bus $j$.

\section{A. Generation and uncontrollable demand dynamics}

We shall consider generation and uncontrollable demand dynamics described by

$$
\begin{gathered}
\dot{p}_{j}^{M}=-\alpha_{j} \omega_{j}, j \in N, \\
d_{j}^{u}=A_{j} \omega_{j}, j \in N,
\end{gathered}
$$

where $A_{j}>0$ and $\alpha_{j} \geq 0$ for all $j \in N$. We assume that there exists at least one bus equipped with the integral controller above, i.e, $\max _{j \in N}\left(\alpha_{j}\right)>0$. In case $\alpha_{j}=0$, the generation output is equal to a constant, namely $p_{j}^{M}=p_{j}^{M, *}$.

Remark 2: The restriction to the class of PI controllers (4) with turbine governor dynamics omited is merely for the sake of simplicity. The extension to high order generation/demand dynamics is reported in [14].

Next, we will consider two classes of decentralized control schemes for discrete loads that provide ancillary services to the power network in the secondary control time-frame and investigate their performance and stability properties. As it will be discussed within the paper, the discrete character of the loads leads to discontinuous system dynamics that can introduce additional complications that need to be explicitly addressed.

\section{LOADS WITH SWITCHING}

\section{A. Problem formulation}

In this section, we consider frequency dependent on-off loads that respond to frequency deviations by switching to an appropriate state in order to aid the network at urgencies. As the network returns to its normal operating conditions, the loads return to their initial state as well, hence affecting users comfort for short periods only. In particular, for each ${ }^{2}$ $j \in N$, we consider the following switching dynamics for the controllable loads:

$$
d_{j}^{c}\left(\omega_{j}\right)=\left\{\begin{aligned}
\bar{d}_{j}, & \omega_{j}>\bar{\omega}_{j} \\
0, & \underline{\omega}_{j}<\omega_{j} \leq \bar{\omega}_{j}, \\
\underline{d}_{j}, & \omega_{j} \leq \underline{\omega}_{j},
\end{aligned}\right.
$$

where $-\infty<\underline{d}_{j} \leq 0 \leq \bar{d}_{j}<+\infty$, and $\bar{\omega}_{j}>0>\underline{\omega}_{j}$. The dynamics in (5) are depicted on Figure 1. Note that these dynamics can be trivially extended to include more discrete values, that would possibly respond to higher frequency deviations.

\footnotetext{
${ }^{2}$ This can be trivially relaxed to any subset of $N$.
} 


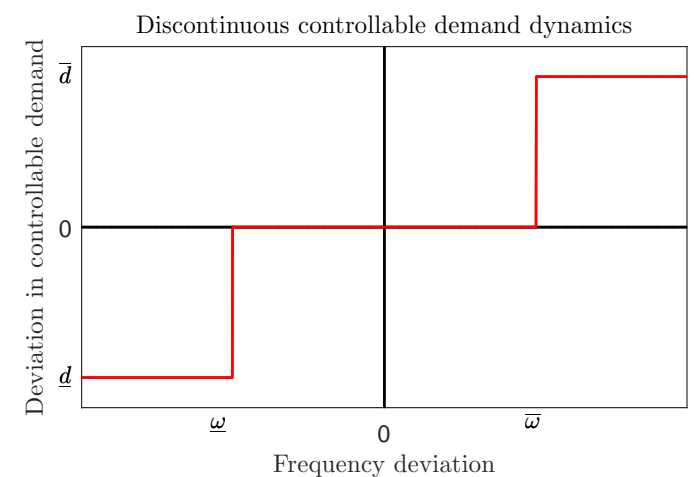

Fig. 1. Switch dynamics for controllable loads as described by (5).

To cope with the switching dynamics of the loads, and to have well-defined solutions to system (5) for all time, we first define a Filippov set valued map as follows:

$$
F\left[d_{j}^{c}\right]=\left\{\begin{array}{l}
\left\{\bar{d}_{j}\right\}, \quad \omega_{j}>\bar{\omega}_{j}, \\
{\left[0, \bar{d}_{j}\right], \quad \omega_{j}=\bar{\omega}_{j}} \\
\{0\}, \quad \underline{\omega}_{j}<\omega_{j}<\bar{\omega}_{j}, \quad j \in N . \\
{\left[\underline{d}_{j}, 0\right], \quad \omega_{j}=\underline{\omega}_{j},} \\
\left\{\underline{d}_{j}\right\}, \quad \omega_{j}<\underline{\omega}_{j},
\end{array}\right.
$$

The state of the interconnected system (3)-(5) is denoted by $x=\left(\eta^{T}, \omega^{T},\left(p^{M}\right)^{T}\right)^{T}$, where any variable without subscript represents a vector with all respective components. For a compact representation of the system, we consider the Filippov set valued map $Q: \mathbb{R}^{n} \rightarrow \mathcal{B}\left(\mathbb{R}^{n}\right)$, with $n=|E|+2|N|$, and write the system dynamics as the following differential inclusion:

$$
\dot{x} \in Q(x)
$$

where

$$
Q(x)=\left\{\begin{array}{l}
\left\{\omega_{i}-\omega_{j}\right\},(i, j) \in E, \\
\left\{\frac { 1 } { M _ { j } } \left(-p_{j}^{L}+p_{j}^{M}-A_{j} \omega_{j}-v_{j}-\sum_{k: j \rightarrow k} p_{j k}\right.\right. \\
\left.\left.+\sum_{i: i \rightarrow j} p_{i j}\right): v_{j} \in F\left[d_{j}^{c}\right]\right\}, j \in N, \\
\left\{-\alpha_{j} \omega_{j}\right\}, j \in N .
\end{array}\right.
$$

Remark 3: As a result of the switching dynamics in (5), the vector field in (3b) will become discontinuous. This discontinuity limits the applicability of classical solutions to the ordinary differential equations (3), and asks for an appropriate notion of solutions. The most suitable notion depends on the objective and the problem at hand. Among several solution notions, see [18], we opt for Filippov solutions [14] which amounts to the relaxation of the differential equation to a differential inclusion, see (7). The idea behind Filippov solutions is to study the behavior of the vector field around a point of discontinuity, and consequently allow the vector field to take any value within an admissible set. As will be observed in Lemma 2, this does not spoil uniqueness of solutions for the dynamics considered in this paper.

\section{B. Equilibria, existence and uniqueness of solutions}

The discontinuous dynamics (5) introduce additional complexity in the analysis of the behavior of (7). First, we study equilibria of the system, and then investigate existence and uniqueness of Filippov solutions. An equilibrium of (7) is defined as follows:

Definition 1: The point $x^{*}=\left(\eta^{*}, \omega^{*}, p^{M, *}\right)$ defines an equilibrium of the system (7) if $0_{n} \in Q\left(x^{*}\right)$.

For an equilibrium of the system, the controllable demand takes its value from a set that depends on $\omega_{j}^{*}$, i.e., $d_{j}^{c, *} \in$ $F\left[d_{j}^{c}\right]\left(\omega_{j}^{*}\right), j \in N$. Lemma 1 below, proven in [14], shows that this set is singleton, namely $Q\left(x^{*}\right)=\left\{0_{n}\right\}$, and $\omega^{*}=$ $\mathbf{0}_{|N|}=d_{j}^{c, *}$.

Lemma 1: For any equilibrium point $x^{*}=\left(\eta^{*}, \omega^{*}, p^{M, *}\right)$ of (7), we have $\omega^{*}=\mathbf{0}_{|N|}$ and $Q\left(x^{*}\right)=\left\{0_{n}\right\}$.

It should further be noted that within the rest of the paper the existence of some equilibrium of (7) is assumed. As evident from Lemma 1, the conditions for existence of an equilibrium can be studied independent of the switching loads, see e.g. [19].

In addition, we impose a constraint on the differences of the phase angles at the equilibrium. This assumption, stated below, is ubiquitous in power network literature, and is treated as a security constraint.

Assumption 1: $\left|\eta_{i j}^{*}\right|<\frac{\pi}{2}$ for all $(i, j) \in E$.

The following lemma, proven in [14], establishes existence and uniqueness of solutions to (3)-(5).

Lemma 2: There exists a unique Filippov solution of (3)-(5) starting from any initial condition $x_{0}=$ $\left(\eta(0), \omega(0), p^{M}(0)\right) \in \mathbb{R}^{n}$.

\section{Stability}

We now state the main result of this section. Its proof can be found in [14].

Theorem 1: Suppose that there exists an equilibrium $\left(\eta^{*}, \omega^{*}, p^{M, *}\right)$ of (7) for which Assumption 1 is satisfied. Then there exists an open neighborhood $\Xi$ of this equilibrium such that Filippov solutions $\left(\eta, \omega, p^{M}\right)$ of (7) starting in this region asymptotically converge to the set of equilibria of the system. In particular, the frequency vector $\omega$ converges to $\omega^{*}=0_{|N|}$.

The theorem above establishes stability of the power network (3)-(4) with on-off load side control (5), and shows that frequency is restored to its nominal value after a transient load-side participation.

\section{Zeno behavior}

A possible feature of switching and hybrid systems is the occurrence of infinitely many switches within some finite time, a phenomenon known as Zeno behavior (e.g. [20]). Such behavior is often undesirable and impedes practical implementations.

In our setting, Zeno behavior may occur in on-off loads as shown numerically in Section VI. The reason such behavior may occur is that the frequency derivative may change sign when passing a discontinuity, causing the vector field to point towards the discontinuity. For instance, suppose that $0<\dot{\omega}_{j}\left(t_{1}\right)<\bar{d}_{j}$ for some time instant $t_{1}>0$, and that the threshold $\bar{\omega}_{j}$ is met at this time. Then, the load $d_{j}^{c}$ switches on, causing a sign change in the value of $\dot{\omega}_{j}$. Hence, the frequency vector field will point at a direction of frequency decrease that will force the load to switch off. These on/off switches occur infinitely many times in a 


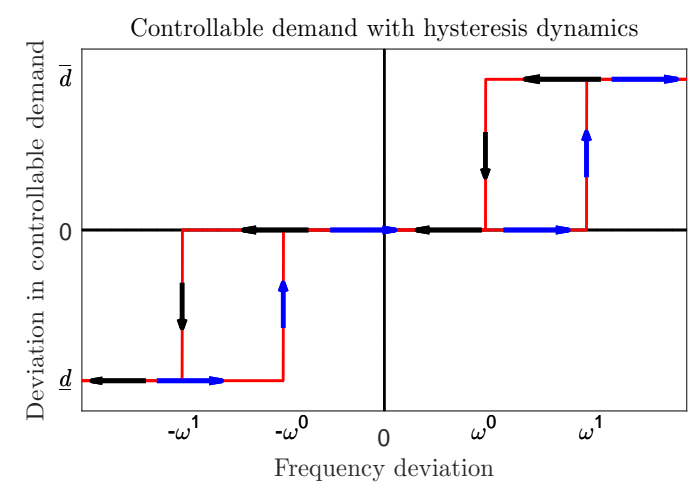

Fig. 2. Hysteresis dynamics for controllable loads as described by (8).

finite time, resulting in the aforementioned Zeno behavior. Note that this phenomenon is only observed here during the transient response of the loads, as the mechanical power injection (4) will eventually dictate the sign of the vector field and regulate the frequency to its nominal value as shown in Theorem 1.

\section{HySTERESIS ON CONTROLLABLE LOADS}

\section{A. Problem formulation}

In this section, we propose the use of hysteretic dynamics in on-off controllable loads, which means that a controllable load switches on and off at different frequency thresholds. As will be observed, this modification will ensure that the system does not exhibit Zeno behavior. For relevant applications of hysteric dynamics in ruling out chattering, Zeno behavior, and other undisered features, see. e.g. [21][23].

We consider the following hysteretic dynamics for controllable loads:

$$
\begin{gathered}
\sigma_{j}^{c}=\bar{d}_{j} \sigma_{j} \\
\sigma_{j}\left(t^{+}\right)= \begin{cases}\operatorname{sgn}\left(\omega_{j}\right), & \left|\omega_{j}\right| \geq \omega_{j}^{1} \\
0, & \left|\omega_{j}\right| \leq \omega_{j}^{0} \\
\sigma_{j}(t), & \text { otherwise }\end{cases}
\end{gathered}
$$

where $j \in N, t^{+}=\lim _{\epsilon \rightarrow 0}(t+\epsilon)$, and the frequency thresholds $\omega_{j}^{0}, \omega_{j}^{1}$, satisfy $\omega_{j}^{1}>\omega_{j}^{0}>0$.

The dynamics described in (8) are depicted in Figure 2. Note that $\sigma_{j}$ takes its value from the set $P=\{-1,0,1\}$.

Now the behavior of the system (3),(4),(8), can be described by the states $z=(x, \sigma)$, where $x=\left(\eta, \omega, p^{M}\right) \in \mathbb{R}^{n}$ is the continuous state, and $\sigma \in P^{|N|}$ the discrete state. The domain of solution is then equal to $\mathbb{R}^{n} \times P^{|N|}$, which we denote in short by $\Lambda$.

The continuous part of the dynamics (3),(4),(8), is given by

$$
\begin{gathered}
\dot{\eta}_{i j}=\omega_{i}-\omega_{j},(i, j) \in E, \\
M_{j} \dot{\omega}_{j}=-p_{j}^{L}+p_{j}^{M}-\left(\bar{d}_{j} \sigma_{j}+A_{j} \omega_{j}\right) \\
-\sum_{k: j \rightarrow k} p_{j k}+\sum_{i: i \rightarrow j} p_{i j}, j \in N, \\
p_{i j}=B_{i j} \sin \eta_{i j}-p_{i j}^{n o m},(i, j) \in E, \\
\dot{p}_{j}^{M}=-\alpha_{j} \omega_{j}, j \in N, \\
\dot{\sigma}_{j}=0, j \in N .
\end{gathered}
$$

This is valid when $z$ belong to the set

$$
C=\left\{z \in \Lambda: \sigma_{j} \in \mathcal{I}_{j}\left(\omega_{j}\right), \forall j \in N\right\}
$$

where

$$
\mathcal{I}_{j}\left(\omega_{j}\right)= \begin{cases}\left\{\operatorname{sgn}\left(\omega_{j}\right)\right\}, & \left|\omega_{j}\right| \geq \omega_{j}^{1}, \\ \{0\}, & \left|\omega_{j}\right| \leq \omega^{0}, \\ \left\{0, \operatorname{sgn}\left(\omega_{j}\right)\right\}, & \omega^{0}<\left|\omega_{j}\right|<\omega^{1} .\end{cases}
$$

Alternatively, when $z \in \Lambda \backslash C$, the system dynamics evolve according to the following discrete update rule:

$$
\begin{aligned}
x^{+} & =x \\
\sigma_{j}\left(t^{+}\right) & = \begin{cases}\operatorname{sgn}\left(\omega_{j}\right), & \left|\omega_{j}\right| \geq \omega_{j}^{1} \\
0, & \left|\omega_{j}\right| \leq \omega_{j}^{0}\end{cases}
\end{aligned}
$$

where the latter is in agreement with (8b). We can now provide the following compact representation for the hybrid system (3),(4),(8),

$$
\begin{gathered}
\dot{z}=f(z), z \in C, \\
z^{+}=g(z), z \in D,
\end{gathered}
$$

where $C$ is given by (10), $D=\Lambda \backslash C$, and $z^{+}=z\left(t^{+}\right)$. The maps $f(z): C \rightarrow \Lambda$ and $g(z): D \rightarrow C$ are given by (9) and (11), respectively. Note that $z^{+}=g(z)$ represents a discrete dynamical system where $z^{+}$is determined by the current value of the state $z$ and the update rule given by $g$.

We assume that the initial conditions are compatible with (12), or essentially with the transition map in (8b). This means that, for each $j \in N, \sigma_{j}(0) \in \overline{\mathcal{I}}_{j}\left(\omega_{j}(0)\right)$ where

$$
\overline{\mathcal{I}}_{j}\left(\omega_{j}\right)= \begin{cases}\left\{\operatorname{sgn}\left(\omega_{j}\right)\right\}, & \left|\omega_{j}\right|>\omega_{j}^{1} \\ \{0\}, & \left|\omega_{j}\right|<\omega^{0} \\ \left\{0, \operatorname{sgn}\left(\omega_{j}\right)\right\}, & \omega^{0} \leq\left|\omega_{j}\right| \leq \omega^{1} .\end{cases}
$$

We write the condition above in vector form as $\sigma(0) \in$ $\overline{\mathcal{I}}(\omega(0))$, and we denote the set of compatible initial conditions by $\Lambda_{0} \subseteq \Lambda$.

\section{B. Analysis of equilibria and solutions}

Before investigating stability of the hybrid system in (12), we characterize its equilibria, and establish existence and uniqueness of solutions.

Note that we call a point $z^{*}=\left(x^{*}, \sigma^{*}\right)$ an equilibrium of (12) if $f\left(z^{*}\right)=0, z^{*} \in C$ or $z^{*}=g\left(z^{*}\right), z^{*} \in D$. Now, we state the following lemma:

Lemma 3: For any equilibrium point $z^{*}=\left(x^{*}, \sigma^{*}\right)$ of (12), we have $\omega^{*}=\sigma^{*}=\mathbf{0}_{|N|}$. Moreover, $z^{*} \in C$.

To proceed further, we need to assume existence of some equilibrium of (12). As evident from Lemma 3, the feasibility of this assumption is independent of the on-off loads and has been studied in literature (e.g. [19]).

We shall borrow the definitions of a hybrid time domain and solution to a hybrid system from [20], [23].

Definition 2: A hybrid time domain is a subset of $\mathbb{R}_{\geq 0} \times$ $\mathbb{N}_{0}$ consisting of, potentially infinite, time intervals of the form $\left[t_{\ell}, t_{\ell+1}\right] \times\{\ell\}$, where $0=t_{0} \leq t_{1} \leq \ldots$, or of finitely many such intervals with the last one possibly of the form $\left[t_{\ell}, t_{\ell+1}\right] \times\{\ell\},\left[t_{\ell}, t_{\ell+1}\right) \times\{\ell\}$ or $\left[t_{\ell}, \infty\right) \times\{\ell\}$. Consider 
a function $z(t, \ell)$ defined on a hybrid time domain $K$ such that for every fixed $\ell, t \rightarrow z(t, \ell)$ is a locally absolutely continuous function on the interval $T_{\ell}=\{t:(t, \ell) \in K)$. The function $z(t, \ell)$ is a solution to (12) if $z(0,0) \in \Lambda_{0}$ and for each $\ell$ it holds that

$$
\begin{aligned}
& \dot{z}(t, \ell)=f(z(t, \ell)), \text { for almost all } t \in T_{\ell}, \\
& z(t, \ell) \in C, \text { for all } \mathrm{t} \in\left[\min T_{\ell}, \sup T_{\ell}\right), \\
& z(t, \ell+1)=g(z(t, \ell)), z(t, \ell) \in D \\
& \text { for all }(t, \ell) \in K \text { such that }(t, \ell+1) \in K .
\end{aligned}
$$

A solution $z(t, \ell)$ is complete if $K$ is unbounded. A solution is maximal if it cannot be extended ${ }^{3}$.

For convenience in the presentation we will refer to maximal solutions by just solutions. Existence and uniqueness of solutions to (12) are established in the following lemma.

Lemma 4: There exists a complete unique solution $z=$ $(x, \sigma)$ to $(12)$, starting from any initial condition $z(0,0) \in$ $\Lambda_{0}$.

Furthermore, the following proposition shows the existence of some finite dwell time between switches of states $\sigma_{j}$ for any bounded solution. Within it, we denote the timeinstants where the value of $\sigma_{j}$ changes by $t_{\ell, j}, \ell \in \mathbb{N}_{0}, j \in N$.

Proposition 1: For any complete bounded solution of (12), there exists $\tau_{j}>0$ such that $\min _{\ell \geq 1}\left(t_{\ell+1, j}-t_{\ell, j}\right) \geq \tau_{j}$ for any $j \in N$.

Remark 4: The importance of Proposition 1 is that it shows that no Zeno behavior will occur for any complete bounded solution of system (12). This is because for any finite time interval $\tau=\min _{j} \tau_{j}, j \in N$, the vector $\sigma$ changes at most $|N|$ times. This highlights the practical advantage of (12) compared to (7). This analytic result is verified by numerical simulations in a realistic power network, as discussed in section VI.

\section{Stability of hysteresis system}

Now, we are at the position to state the stability result concerning the system (12).

Theorem 2: Let $z^{*}=\left(x^{*}, \sigma^{*}\right)$, with $\omega^{*}=\sigma^{*}=0_{|N|}$, be an equilibrium of (12) for which Assumption 1 holds. Then there exists an open neighborhood $S$ of $x^{*}$ such that solutions $z=(x, \sigma)$, with $x(0) \in S$ and $\sigma(0) \in \overline{\mathcal{I}}(\omega(0))$, asymptotically converge to the set of equilibria of (12). In particular, the vectors $\omega$ and $\sigma$ converge to the vector $0_{|N|}$.

Theorem 2 shows that the hysteretic dynamics in (8) do not compromise the stability of the system. This, together with the absence of Zeno behavior shown in Proposition 1 , promotes the use of hysteretic dynamics as a means to provide practical and non-disruptive on-off load side control to the power network.

\section{Simulation on the IEEE 68-BUS System}

In this section we simulate the IEEE New York/ New England 68-bus interconnection system using the Power System Toolbox [24] to numerically verify our analytic results. The simulated model is more detailed and realistic

\footnotetext{
${ }^{3}$ That is, there is no other solution $\tilde{z}$ with time domain $\tilde{K}$ such that $K$ is a proper subset of $\tilde{K}$ and $\tilde{z}$ agrees with $z$ on $K$.
}

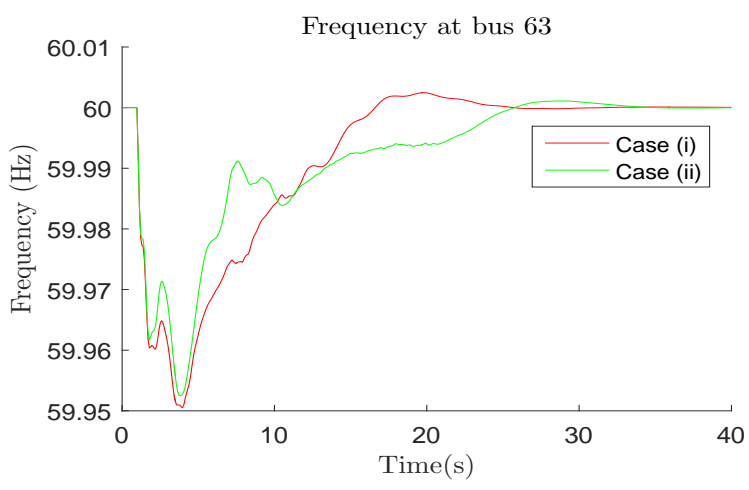

Fig. 3. Frequency at bus 103 with controllable load dynamics as in the following two cases: i) Switching case, ii) Hysteresis case.

than our analytical one, including line resistances, a DC12 exciter model and power system stabiliser ${ }^{4}$.

The considered system consists of 52 load buses and 16 generation buses and has a total real power of $16.41 \mathrm{GW}$. For our simulation, we added five uncontrollable loads on units $2,8,9,16$ and 17, each having a step increase of magnitude 1 p.u. (base $100 \mathrm{MVA}$ ) at $t=1$ second.

Within the simulations, we considered generators with dynamics as described by (4a) at all generation buses and also controllable demand on 30 load buses, with loads controlled every $10 \mathrm{~ms}$.

The system was tested at two different cases. In case (i), we considered on-off controllable loads on 30 load buses with dynamics as in (5). The values for $\bar{\omega}_{j}$ were selected from a uniform distribution within the range [0.02 0.07] and those of $\underline{\omega}_{j}$ by $\underline{\omega}_{j}=-\bar{\omega}_{j}$. For case (ii) controllable loads were also included on 30 load buses, but with dynamics described by (8). We used the same frequency thresholds for both cases for a fair comparison, with $\omega_{j}^{1}=\bar{\omega}_{j}$ and $\omega_{j}^{0}=\omega_{j}^{1} / 4$. Also, $\bar{d}=-\underline{d}=0.2 p$.u. was used for both cases. Cases (i) and (ii) will be referred as the 'switching' and 'hysteresis' cases respectively.

The frequency at bus 63 for the two cases considered is depicted in Fig. 3 which demonstrates that frequency returns to its nominal value for both cases, as suggested in Theorems 1 and 2. Moreover, Fig. 4 shows that on-off loads are able to cause significant reduction in the maximum frequency overshoot, by comparing the largest deviation in frequency with and without on-off controllable loads at all buses. Figure 5 demonstrates very fast switches of controllable loads at 4 buses for case (i), indicating Zeno behaviour. In comparison, such behaviour is not observed when case (ii) is considered, as shown in Figure 6 which verifies that hysteresis eliminates Zeno behavior at controllable loads, as suggested by the analysis in this paper. The 4 selected buses on Figures 5 and 6 were those with the minimum time between consecutive switches in the hysteretic loads of case (ii). Moreover, both figures depict times up to $25 \mathrm{~s}$ since all loads remain switched off afterwards. Note that the fact that all loads return to their nominal demand after a brief period demonstrates the nondisruptive nature of the two schemes.

\footnotetext{
${ }^{4}$ The details of the simulation models can be found in the Power System Toolbox data file datanp48.
} 


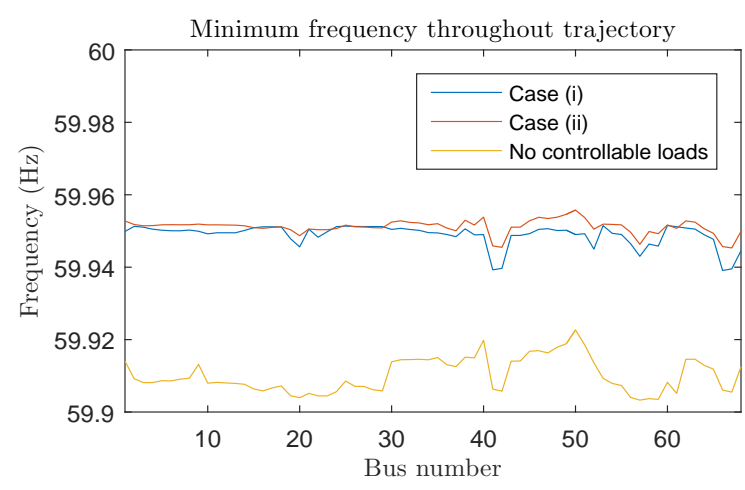

Fig. 4. Largest frequency overshoot for buses $1-40$ for three cases: (i) Use of switching loads, (ii) Use of hysteresis loads, (iii) No use of controllable loads. Note that the graphs for cases (i), (ii) are almost identical and indistinguishable in the figure.
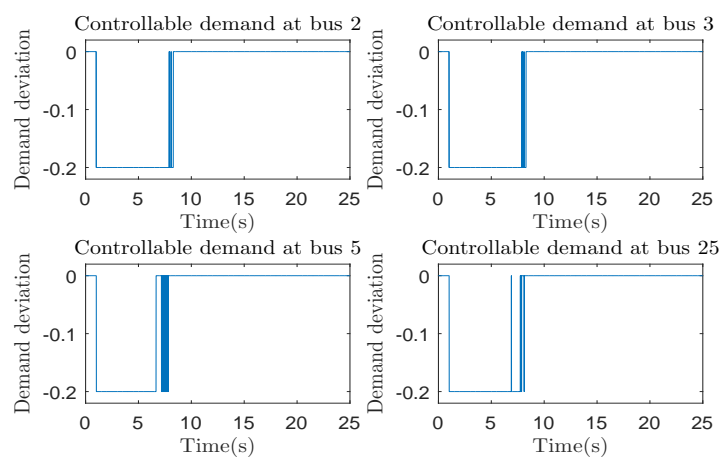

Fig. 5. Controllable demand deviations at 4 buses with Switching on-off loads.
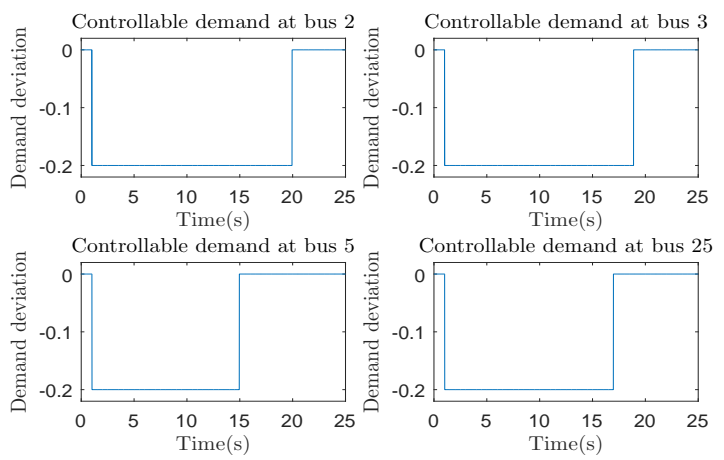

Fig. 6. Controllable demand deviations at 4 buses with Hysteresis on-off loads.

\section{CONCLUSION}

We have considered the problem of secondary frequency control where controllable on-off loads provide ancillary services. We first considered loads that switch on when some frequency threshold is reached and off otherwise. Stability guarantees are provided for such loads. Furthermore, it is discussed that such schemes might exhibit arbitrarily fast switching, which might limit their practicality. To cope with this issue, on-off loads with hysteretic dynamics were considered. It has been shown that such loads do not exhibit any Zeno behavior and that their inclusion does not compromise power network stability. Hence, such schemes are usable for practical implementations. Both schemes ensure that controllable loads return to their nominal behavior at equilibrium and hence that disruptions occur for brief periods only. Our analytic results have been verified with numerical simulations on the IEEE 68-bus New York/New England system where it was shown that the presence of on-off loads reduces the frequency overshoot and that hysteresis schemes resolve issues caused by Zeno-like behavior. Interesting potential extensions in the analysis include more advanced on-off load dynamics as well as incorporating voltage dynamics.

\section{REFERENCES}

[1] H. Lund, "Large-scale integration of optimal combinations of pv, wind and wave power into the electricity supply," Renewable energy, vol. 31, no. 4, pp. 503-515, 2006.

[2] A. Ipakchi and F. Albuyeh, "Grid of the future," IEEE power and energy magazine, vol. 7, no. 2, pp. 52-62, 2009.

[3] P. Kundur, N. J. Balu, and M. G. Lauby, Power system stability and control, vol. 7. McGraw-hill New York, 1994.

[4] A. Ulbig, T. S. Borsche, and G. Andersson, "Impact of low rotational inertia on power system stability and operation," IFAC Proceedings Volumes, vol. 47, no. 3, pp. 7290-7297, 2014.

[5] A. Molina-Garcia, F. Bouffard, and D. S. Kirschen, "Decentralized demand-side contribution to primary frequency control," IEEE Transactions on Power Systems, vol. 26, no. 1, pp. 411-419, 2011.

[6] S. Trip and C. De Persis, "Optimal generation in structure-preserving power networks with second-order turbine-governor dynamics," in European Control Conference (ECC), pp. 916-921, IEEE, 2016.

[7] C. Zhao, U. Topcu, N. Li, and S. Low, "Design and stability of loadside primary frequency control in power systems," IEEE Transactions on Automatic Control, vol. 59, no. 5, pp. 1177-1189, 2014.

[8] A. Kasis, E. Devane, C. Spanias, and I. Lestas, "Primary frequency regulation with load-side participation Part I: stability and optimality," IEEE Transactions on Power Systems, vol. 32, no. 5, pp. 3505-3518, 2017.

[9] E. Devane, A. Kasis, M. Antoniou, and I. Lestas, "Primary frequency regulation with load-side participation Part II: Beyond passivity approaches," IEEE Transactions on Power Systems, vol. 32, no. 5, pp. 3519-3528, 2017.

[10] E. Mallada, C. Zhao, and S. Low, "Optimal load-side control for frequency regulation in smart grids," in 52nd Annual Allerton Conference on Communication, Control, and Computing (Allerton), pp. 731-738, IEEE, 2014.

[11] S. Trip, M. Bürger, and C. De Persis, "An internal model approach to (optimal) frequency regulation in power grids with time-varying voltages," Automatica, vol. 64, pp. 240-253, 2016.

[12] A. Kasis, E. Devane, and I. Lestas, "Stability and optimality of distributed schemes for secondary frequency regulation in power networks," in 55th IEEE Conference on Decision and Control (CDC), pp. 3294-3299, IEEE, 2016.

[13] T. Liu, D. J. Hill, and C. Zhang, "Non-disruptive load-side control for frequency regulation in power systems," IEEE Transactions on Smart Grid, vol. 7, no. 4, pp. 2142-2153, 2016.

[14] A. Kasis, N. Monshizadeh, and I. Lestas, "Secondary frequency control with on-off load side participation in power networks," arXiv preprint arXiv:1708.09351, 2017.

[15] T. Tao, An introduction to measure theory, vol. 126. American Mathematical Soc., 2011.

[16] J. Cortes, "Discontinuous dynamical systems," IEEE Control Systems, vol. 28, no. 3, pp. 38-73, 2008.

[17] A. R. Bergen and V. Vittal, Power Systems Analysis. Prentice Hall, 1999.

[18] S. Hu, "Differential equations with discontinuous right-hand sides," Journal of Mathematical Analysis and Applications, vol. 154, no. 2, pp. 377-390, 1991.

[19] F. Dörfler, M. Chertkov, and F. Bullo, "Synchronization in complex oscillator networks and smart grids," Proceedings of the National Academy of Sciences, vol. 110, no. 6, pp. 2005-2010, 2013.

[20] R. Goebel, R. G. Sanfelice, and A. R. Teel, "Hybrid dynamical systems," IEEE Control Systems, vol. 29, no. 2, pp. 28-93, 2009.

[21] H. Lee and V. I. Utkin, "Chattering suppression methods in sliding mode control systems," Annual reviews in control, vol. 31, no. 2, pp. 179-188, 2007.

[22] C. G. Mayhew, R. G. Sanfelice, and A. R. Teel, "Quaternion-based hybrid control for robust global attitude tracking," IEEE Transactions on Automatic Control, vol. 56, no. 11, pp. 2555-2566, 2011.

[23] F. Ceragioli, C. De Persis, and P. Frasca, "Discontinuities and hysteresis in quantized average consensus," Automatica, vol. 47, no. 9, pp. 1916-1928, 2011.

[24] K. Cheung, J. Chow, and G. Rogers, "Power system toolbox, v 3.0," Rensselaer Polytechnic Institute and Cherry Tree Scientific Software, 2009. 